\title{
MAGNETIC BRAKING IN CATACLYSMIC AND LOW-MASS
}

\section{X-RAY BINARIES*}

\author{
O. VILHU** \\ Nordita, Copenhagen, Denmark
}

(Received 22 July, 1983)

\begin{abstract}
The chromospheric-coronal emission of lower Main-Sequence single and binary stars can be correlated with an activity parameter of type $R=g(B-V) P^{-1}$, where $P$ is the rotation or orbital period and $g(B-V)$ a function of the color resembling the convective turnover time. Observations indicate that the active region area coverage filling factor grows as $R^{2}$, and the whole stellar surface becomes filled with closed loop structures at $R \approx 3$. A braking formula is proposed (Equation 4 ) to include all periods $\left(0.1 \leqslant R \leqslant 30^{d}\right)$ and spectral types $\mathbf{F}-\mathbf{M}$. On the basis of this equation, the mass transfer rates in compact binaries (driven by the gradual loss of orbital angular momentum) are discussed. It is concluded that the magnetic braking has good chances of being that mechanism which drives the mass transfer in cataclysmic variables and galactic bulge $\mathrm{X}$-ray sources.
\end{abstract}

\section{Introduction}

In cataclysmic variables (CV) and galactic bulge X-ray sources (bursters), the mass exchange is often much higher than predicted by gravitational radiation alone. This applies to models where a low mass Main-Sequence star fills its Roche lobe and is forced to lose mass onto the compact companion by gradual loss of angular momentum.

Rappaport et al. (1982) have shown that the mass exchange rate due to radiation of gravitational waves is constrained to a value lower than $2 \times 10^{-10} M_{\odot} \mathrm{yr}^{-1}$, while the rate for many CV's exceeds this limit by one hundred times (Tutukov and Yungelson, 1979). Verbunt and Zwaan (1981) give a similar number to power the brightest galactic bulge $\mathrm{X}$-ray sources, if the model by Joss and Rappaport (1979) is accepted where a neutron star is accompanied by a low-mass $\left(<0.8 M_{\odot}\right)$ Roche-lobe filling Main-Sequence star. For this reason, other mechanisms besides the gravitational radiation, have been searched for.

Verbunt and Zwaan (1981) and Tutukov (1983) extrapolated the empirical braking law by Skumanich (1972): $V_{\mathrm{eq}}=f 10^{14} t^{-0.5} \mathrm{~cm} \mathrm{~s}^{-1}$, where $f$ is a constant of the order of unity. This leads to the angular momentum loss rate of the form $\mathrm{d} J / \mathrm{d} t \sim f^{-2} P^{-3}$, where $P$ is the binary period. Applying this law for the synchronously co-rotating cool secondary in a compact binary, they found mass transfer rates of the order $10^{-8}-10^{-9} M_{\odot} \mathrm{yr}^{-1}$.

Spruit and Ritter (1983) used implicitly the same idea, and in particular

\footnotetext{
* Paper presented at the Lembang-Bamberg IAU Colloquium No. 80 on Double Stars: Physical Properties and Generic Relations, held at Indonesia, 3-7 June, 1983.

** On leave from Observatory and Astrophysics Laboratory, University of Helsinki.
} 
explained the $2^{\mathrm{h}}-3^{\mathrm{h}}$ gap in CV's by a sudden decrease in the braking when then secondary becomes fully convective at around $0.3 M_{\odot}$ (having some observational support).

Strictly speaking, Skumanich's law applies for relatively slowly rotating solar-type stars only $\left(P>3^{\mathrm{d}}\right)$, and its extrapolation to over 10 times shorter periods and much later spectral types may be dangerous. In particular, Vilhu (1982) showed that the best explanation for the close binary period statistics and for contact binary production is achieved if the exponent of $P$ in the angular momentum loss formula changes from -3 to -1.5 when $P<3$ days. Rucinski (1983) reached a similar conclusion by studying the period distribution of a sample of close detached binaries.

Further, although the time dependence $\left(t^{-0.5}\right)$ may be independent of the spectral type (see Skumanich and Eddy, 1981), the constant $f$ may well have a strong dependence on it.

In this colloquium, I will present some recent results on chromospheric-coronal activity of Main-Sequence stars (Section 2) and discuss the braking law in this context (Section 3).

\section{The Activity Parameter}

Noyes (1982) suggested the use of an activity parameter of type

$$
R=g(B-V) P^{-1}
$$

for Main-Sequence stars of spectral types G-K. Vilhu (1983) used a similar parameter and extended the function $g(B-V)$ to also include F-stars. This function, shown in Figure 1, was constructed to obtain the best correlations between $R$ and fractional fluxes $f / f_{\text {bol }}$ from the chromospheric-coronal plasma.

If the function $g(B-V)$ is interpreted as the convective turnover time, then $R$ is equal to the inverse Rossby number or to the square root of the dynamo number (Durney and Latour, 1978).

Figure 2 shows fractional $\mathrm{Ca} \mathrm{K}+\mathrm{H}$ fluxes for single Main-Sequence stars vs the activity parameter, as reproduced from Figures 4 and 5 of Noyes (1982), and supplemented with binary star observations taken from Middelkoop (1981) and then reduced to the fractional fluxes $f / f_{\text {bol }}$. Figures 3-5 show similar correlations for hotter plasma levels, as observed with IUE and Einstein satellites (for details and references see Vilhu, 1983).

Striking features of these diagrams are the increasing slopes when the emitting plasma temperature increases (CHR $\rightarrow$ TR $\rightarrow$ COR) and the 'saturation' observed at $R \equiv R_{c} \approx 3$, corresponding roughly to the period $P=3$ days for G2-stars. Binary and single stars seem to follow the same trend, and for $R<3$, the binary effects (apart from the synchronism) seem to be small. The lowest point in Figure 2 is a binary (HD $92168, P=7 \mathrm{~d} 8, \mathrm{~F} 8 \mathrm{~V}$ ). It deviates mostly from the general trend, 


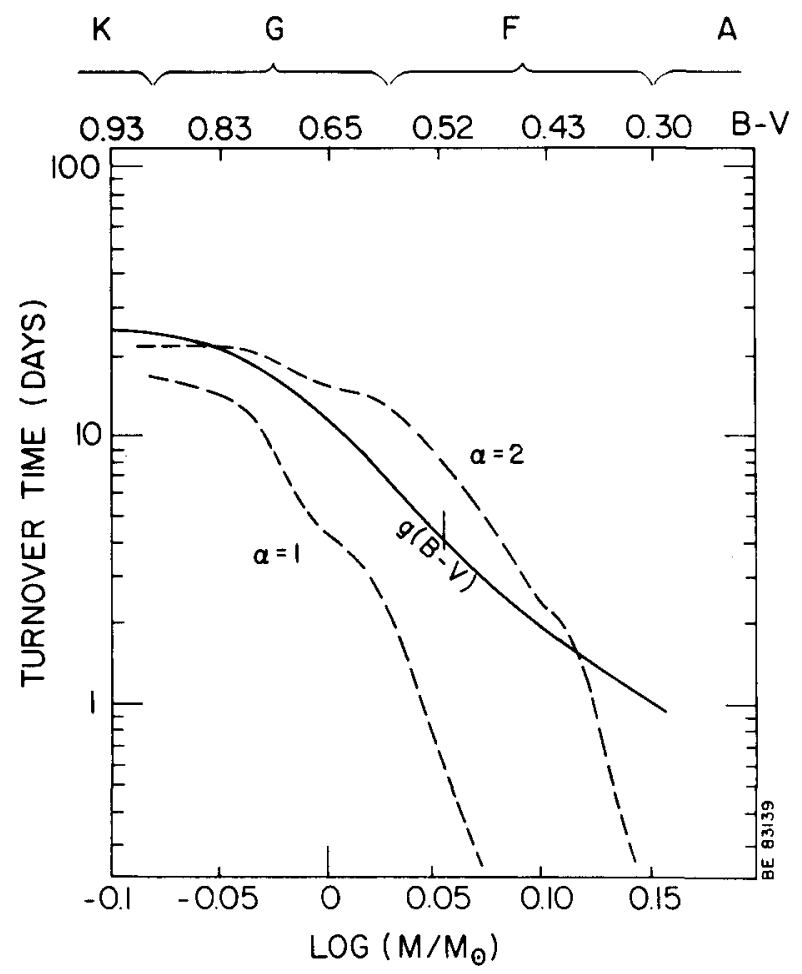

Fig. 1. The function $g(B-V)$ used to compute the activity parameter $R$. For comparison, the convective turnover time curves for mixing lengths $\alpha=1$ and $\alpha=2$ are shown as taken from Gilman (1980). The stellar masses and spectral types are marked. To the left from the small vertical bar, $g(B-V)$ is the same as in Noyes (1982).

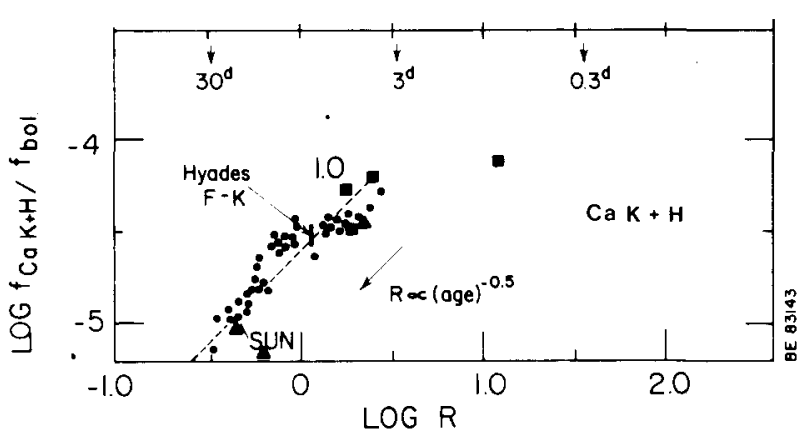

Fig. 2. Calcium $\mathrm{K}+\mathrm{H}$-line fluxes (scaled with the bolometric flux) versus the activity parameter $R$ for single Main-Sequence stars (dots) as taken from Noyes (1982). Binary star observations (filled squares and triangles) are from Middelkoop (1981) and reduced further to fractional fluxes $f / f_{\text {bol }}$. The vertical bar shows the range for Hyades stars between $0.5<B-V<1.0$ (taken from Noyes, 1982 and reduced to $\left.f / f_{\text {bol }}\right)$. The arrow shows the direction of the time-evolution for a single star. The rotation period for a G2 star (in days) is marked. For the meaning of the symbols see also Figures 4 and 5. 


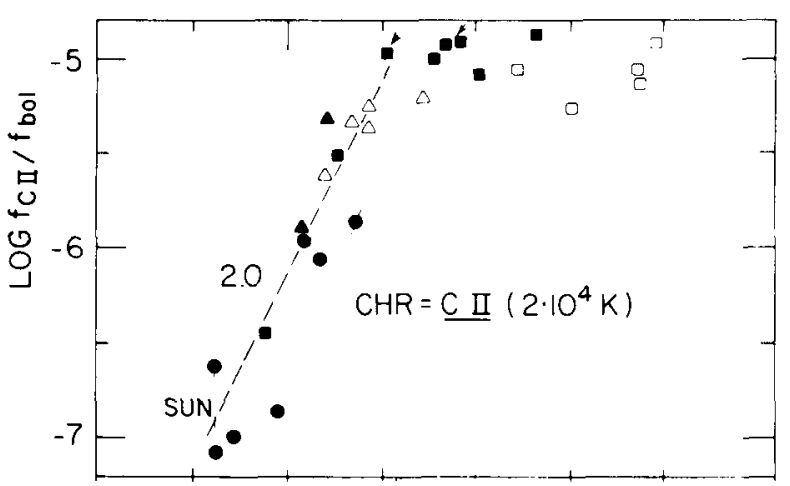

Fig. 3. The fractional $C_{11} 1335$ fluxes vs the activity parameter $R$. The symbols used are explained in Figures 4 and 5 .

indicating possible departure from synchronism which generally may be assumed to be valid at periods $P \leqq 9^{\text {d }}$ (see, e.g., Middelkoop, 1981). Thus a synchronized binary seems to produce the same chromospheric-coronal fluxes as a single star with the same rotation rate and spectral type (at least for $R<3$ ).

The question of extra binary effects for $R>3$ remains open, since there does not exist known single Main-Sequence stars in this domain. Close proximity effects are, however, clearly seen in the coronal emission of contact binaries (see Figure 5).

Vilhu (1983) interpreted the slopes in Figures 2-5 assuming a two-component

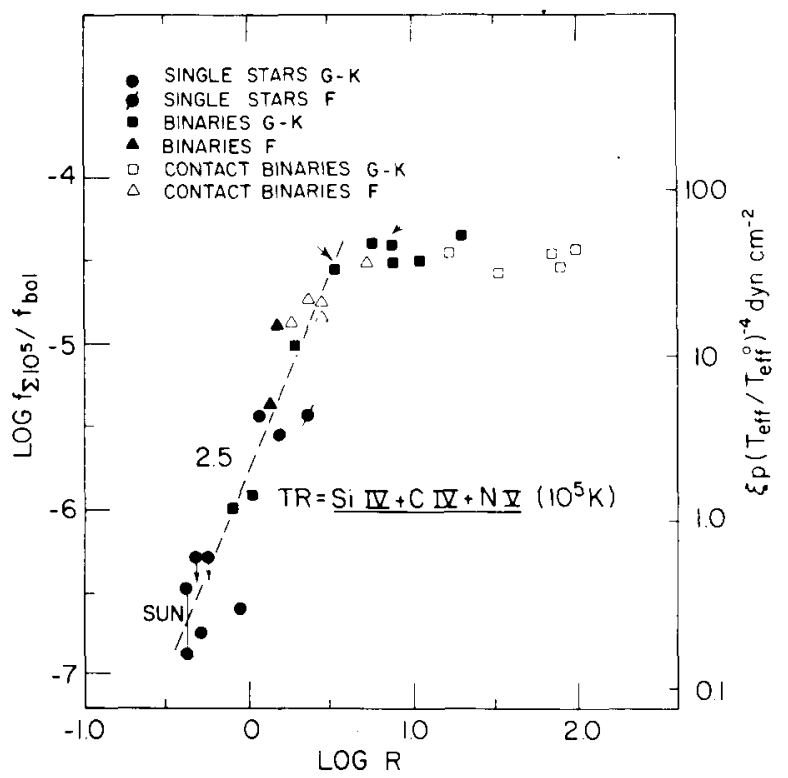

Fig. 4. The same as Figure 3, but for $\mathrm{N} v 1240+\mathrm{C}$ Iv $1549+\mathrm{Si}$ IV 1400 . The right vertical scale gives the values for mean loop pressures (for details, see Vilhu, 1983). 


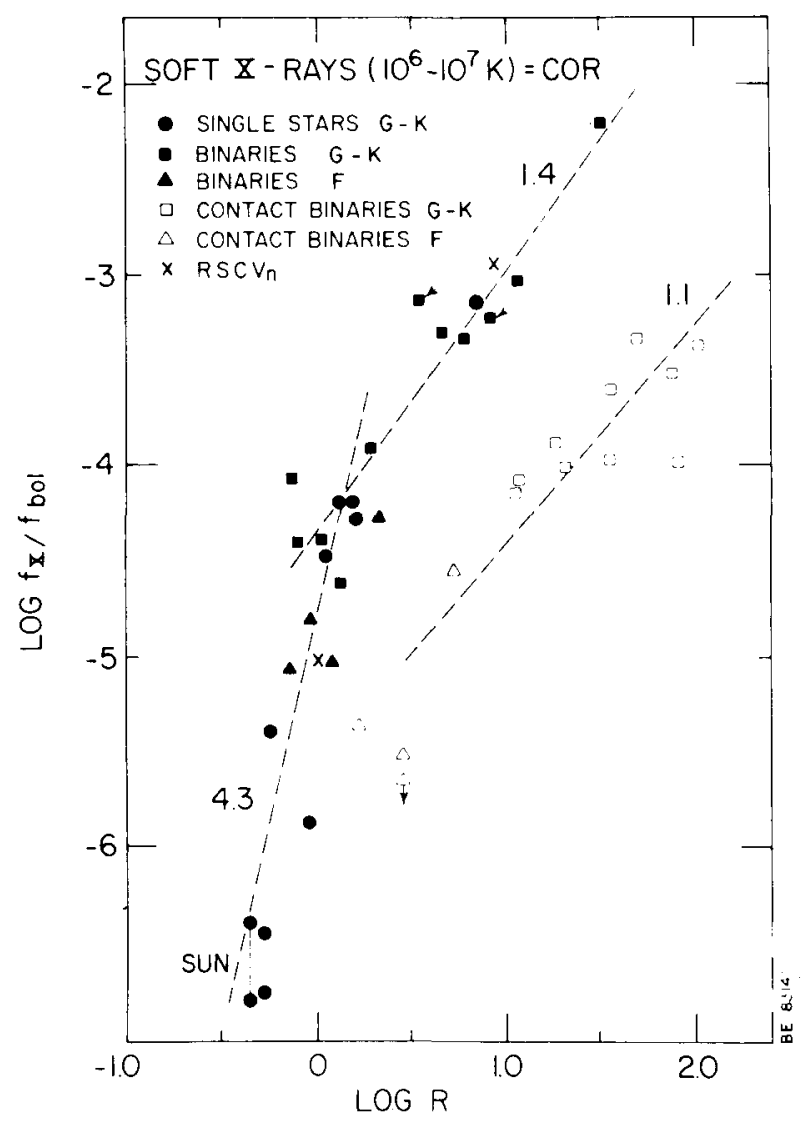

Fig. 5. The same as Figure 3, but for soft X-rays $(0.2-4 \mathrm{keV})$. The two crosses show the domain for RSCVn stars if the activity parameter $R$ is further multiplied by (gravity) ${ }^{-0.25}$ (for details see Vilhu, 1983). The numbers in Figures $3-5$ give the slopes of $\log f / f_{\text {bol }}$ vs $\log R$.

stellar surface (quiet plus active regions) where the active region filling factor grows as $\propto R^{2}$ (for $R<3$ ). Since the dynamo number is also proportional to $R^{2}$ (if $g(B-V)$ is interpreted as the convective turnover time) this means that the filling factor is proportional to the dynamo number. The steeper slopes in TR-and COR-fluxes can be explained by increasing coronal loop pressures and sizes with growing $R$. Lower $\mathrm{X}$-rays from contact binaries is probably due to their smaller photospheric twisting velocities.

\section{Magnetic Braking}

The relatively good correlations of Figures $2-5$ suggest that single Main-Sequence stars with $R \lesssim 3(P>3$ d for G2-stars) move down along these diagrams when aging. Skumanich and Eddy (1981) suggest that the common braking law for spectral types $\mathrm{F}-\mathrm{K}$ is of the form $\omega=h(B-V) t^{-0.5}$, where $\omega$ is the angular velocity 
$\left(P^{-1}\right)$ and $h(B-V)$ an unknown function of the colour. We give here some evidence that $h(B-V) \sim g(B-V)^{-1}$, where $g(B-V)$ is the 'turnover time' function in Equation (1) and Figure 1.

If indeed $h(B-V) \propto g(B-V)^{-1}$, then stars at a fixed age should have the same $R$-value (independent of the spectral type), since then $R \equiv g(B-V) \omega \propto t^{-0.5}$, independently of $B-\mathrm{V}$. In this way, each stellar cluster should have its own characteristic $R$-value, the larger one the younger the cluster is.

That this seems to be the case at least for the Hyades cluster (age $5 \times 10^{8} \mathrm{yr}$ ) for $0.5<B-V<1.0$ is demonstrated in Figure 2, where $\mathrm{Ca} \mathrm{K}+\mathrm{H}$ observations of Hyades stars (the thick bar) given by Noyes (1982) are shown after being converted into $f_{\mathrm{CaK}+\mathrm{H}} / f_{\mathrm{bol}}$. The bar is placed at a mean Hyades $R$-value $\left(R_{\mathrm{Hya}} \simeq 1.25\right)$. If the Hyades stars follow $f_{\mathrm{CaK}+\mathrm{H}} / f_{\mathrm{bol}} \propto R$ (as can be reasonably assumed), this result means that the color dependence of $R$ is very small for a fixed age. Catalano and Marilli (1983) find that in Hyades and Pleiades clusters the calcium-luminosity depends on mass roughly as $L_{\mathbf{K}} \propto M^{5}$. This is not very different from the mass-luminosity relation in lower Main-Sequence, from which it follows that $f_{\mathrm{CaK}} / f_{\text {bol }}$ is rather insensitive on stellar mass in Hyades and Pleiades.

This evidence suggests the use of a braking law of the form

$$
R \propto t^{-0.5} \text { or } V_{\mathrm{eq}} \propto r g(B-V)^{-1} t^{-0.5}
$$

where $r$ is the stellar radius, the function $g(B-V)$ is shown in Figure $1, V_{\mathrm{eq}}$ is the equatorial velocity, and $t$ is the age. This braking law allows us to write for the momentum loss ( $J$ is the spin angular momentum)

$$
\frac{1}{J} \frac{\mathrm{d} J}{\mathrm{~d} t} \propto R^{2}
$$

This means (in our interpretation) that $1 / J(\mathrm{~d} J / \mathrm{d} t)$ is proportional to the dynamo number or to the active region filling factor.

Comparing this braking law with that used by Verbunt and Zwaan (1981), one finds easily that the mass transfer rates should be multiplied by a factor of $\dot{M} / \dot{M}_{\mathrm{VZ}}=\left(g(B-V) / g_{\odot} r_{\odot} / r^{2}\right.$, where, $\dot{M}_{\mathrm{VZ}}$ is the rate computed by Verbunt and Zwaan. For a $\mathrm{CV}$ with a $0.3 M_{\odot}$ secondary (corresponding roughly to a period of $3 \mathrm{hr}$ ) this would mean nearly one hundred times larger mass-transfer rates than predicted with $V_{\text {eq }}=f 10^{14} t^{-0.5}$, where $f=$ const.

On the other hand, this extra gain in the mass-transfer rate may be lost by a possible change in the exponent of Equation (3) after the saturation point $R \simeq 3$. Hence, we propose to use a generalized braking law of the type

$$
\frac{1}{J} \frac{\mathrm{d} J}{\mathrm{~d} t} \propto\left(R / R_{c}\right)^{\alpha},
$$

where $\alpha=2$ for $R \leqslant R_{c} \simeq 3$ and $\alpha$ is still unknown for $R>R_{c}$, but the results by Vilhu (1982) and Rucinski (1983) suggest that $\alpha \simeq 0.5$ for $R>R_{c}$. The constant of 
proportionality in this relation is the same for all spectral types F-M and rotational periods $P \lesssim 30^{\mathrm{d}}$.

The simplest magnetic braking theory with a co-rotating wind inside the Alfvén radius predicts $J^{-1} \mathrm{~d} J / \mathrm{d} t \propto B_{0}^{2}$, where $B_{0}$ is the surface magnetic field (see e.g., Durney and Latour, 1978). This law is the same as Equation (4) for $R \lesssim 3$ provided that the mean surface magnetic field is proportional to $R$, as Figure 2 and the linear connection between local solar magnetic fields and $\mathrm{CaK}$ emission suggest (Frazier, 1970).

For $R>\mathrm{R}_{c}(\approx 3)$, however, something qualitatively new takes place in the chromospheric-coronal radiative losses. It is clear, however, that at present very little can be predicted of the behaviour of open field lines (responsible for the braking) on the basis of the closed ones (responsible for the upper chromospherecoronal emission).

A simple and straightforward interpretation of the saturation in Figures 2-5 might lead to the assumption that $\alpha \rightarrow 0$ [in Equation (4)] when $R \rightarrow 3$. However, although the stellar surface may be totally covered with closed field regions at $R>3$ (allowing, seemingly, no place for open field lines), some closed field lines may well open up and allow the braking to increase $(\alpha>0)$ even at $R>3$.

The effect of $\alpha$ on the numerical computations by Verbunt and Zwaan (1981) can be estimated. Assuming a braking law [Equation (4)] with $\alpha=2$ for $R<3$ and $\alpha=0.5$ for $R>3$, one easily computes how much smaller mass transfer rate a $\mathrm{CV}$ with a $3 \mathrm{hr}$ orbital period would have as compared with the case where $\alpha=2$ everywhere. The result is roughly $1 / 100$, and this would then completely cancel the gain due to the lowering of the secondary's mass as discussed above. This estimate shows that the results by Verbunt and Zwaan (1981) and Tutukov (1983) have good chances of being correct.

As mentioned previously, the value 0.5 for $\alpha$ is exactly what can be deduced frem close binary statistics (Vilhu, 1982; Rucinski, 1983), and so far represents the best guess.

One observational test for the parameter $\alpha$ might be accurate long-term $(\mathrm{O}-\mathrm{C})$ observations of very close (but detached) binaries like ER Vul (G0V+G5V, $P=0.7$ days $)$ and UV Leo $(\mathrm{G} 0 \mathrm{~V}+\mathrm{G} 1 \mathrm{~V}, P=0.6$ days). For these systems we predict a systematic decrease of the orbital period by amounts $\mid P / \dot{P} \simeq-10^{9}$ yr and $P / \dot{P} \approx-10^{8}$ yr for $\alpha=0.5$ and 2.0 , respectively.

Observations of stars of different spectral types and ages (in clusters) would also be important to check whether each cluster indeed has its own characteristic $R$ value, as the Hyades data suggest (Figure 2).

\section{References}

Durney, B. R. and Latour, J.: 1978, Geophys. Astrophys. Fluid Dyn. 9, 241.

Catalano, S. and Marilli, E.: 1983, Astron. Astrophys. 121, 190.

Frazier, E. N.: 1970, Solar Phys. 14, 89. 
Gilman, P. A.: 1980, in D. Gray and J. Linsky (eds.), Stellar Turbulence, Springer, Berlin, p. 19.

Joss, P. C. and Rappaport, S.: 1979, Astron. Astrophys. 71, 217.

Middelkoop, F.: 1981, Astron. Astrophys. 101, 295.

Noyes, R. W.: 1983, in J. O. Stenflo (ed.), 'Solar and Stellar Magnetic Fields: Origins and Coronal Effects', IAU Symp. 102, 133.

Rappaport, S., Joss, P. C., and Webbink, R.: 1982, Astrophys. J. 254, 616.

Rucinski, S. M.: 1983 (preprint).

Skumanich, A.: 1972, Astrophys. J. 171, 565.

Skumanich, A. and Eddy, J.: 1981, in R. M. Bonnet and A. K. Dupree (eds.), Solar Phenomena in Stars and Stellar Systems, D. Reidel Publ. Co., Dordrecht, Holland, p. 349.

Spruit, H. C. and Ritter, H.: 1983, Astron. Astrophys. 142, 267.

Tutukov, A. V.: 1983, (preprint).

Tutukov, A. V. and Yungelson, L. R.: 1979, Acta Astron. 25, 665.

Verbunt, F. and Zwaan, C.: 1981, Astron. Astrophys. 100, L7.

Vilhu, O.: 1982, Astron. Astrophys. 109, 17.

Vilhu, O.: 1983. NORDITA preprint 83/21 (Astron. Astrophys., in press). 